\title{
Modelling and control of growing slugs in horizontal multiphase pipe flows II
}

\author{
STEINAR M. ELGS $\nRightarrow T E R * \dagger$
}

Keywords: Active slug control, growing slugs, slugs in horizontal pipelines, slug length observer, multiphase flow, multiphase modeling

In this paper, the use of active control to restrict the length of growing slugs in horizontal pipelines is investigated. Specifically, the paper attempts to determine if such control can be attained with realistic measurements and actuators. Simulations in OLGA2000 show that a feedback controller can use measurements or estimates of slug length to control the growth of a slug in a horizontal pipeline by partially closing inlet or outlet chokes. A control-volume approach is used to develop a low-order model of inlet choke-slug growth dynamics based on mass- and impulse balances. The resulting model is a system of nonlinear differential-algebraic equations, which is suitable for observer-design. The tuned model is found to be in good agreement with experiments and OLGA2000-simulations. Linearizations of the model are found to be observable around realistic trajectories when rates and pressures at the inlet and outlet are measured. An extended Luenberger-observer is shown to give good estimates of slug length and -position in simulations even under model uncertainty.

\section{Introduction}

In offshore petroleum production well fluids in different phases are transported long distances through a single pipeline from wells to processing facilities. These flows consisting of a combination of oil, water, sand and natural gas are referred to as multiphase flow.

One phenomenon which can occur in multiphase flows is slugging, see Figure 1, an intermittent flow pattern where cylinders of liquid called slugs travel through the pipeline separated by areas of stratified flow. Depending on the cause of slugging, the slug length can vary greatly. Short, hydrodynamic slugs do not represent an operational problem, but longer slugs are unwanted for several reasons. Long slugs produce oscillating flow conditions which put strain on equipment. Large variations in flow can also result in tripping or shutdown of the receiving facilities. More importantly, long slugs significantly increase the pressure drop over the pipeline, thereby reducing production rates (Godhavn, Fard \& Fuchs, 2005).

Terrain-induced slugging can occur in petroleum production in flowline-riser pipelines which transport well fluids from the sea floor to topside production facilities. The idea of using active control of topside chokes to suppress terrain-induced slugging has been around for almost thirty years (Schmidt, Brill \& Beggs, 1979), and many implementations exist (Skofteland \& Godhavn, 2003; Havre \& Dalsmo, 2001).

A type of long slugs which has attracted far less attention from researchers is long, growing slugs in horizontal pipelines. Extremely long, constantly growing slugs with

*Corresponding author.

$\dagger$ Department of Engineering Cybernetics, Norwegian University of Science and Technology, Trondheim, Norway Steinar.Elgsater@itk.ntnu.no

IIAn early version of this paper was presented at SIMS 2005, the 46th Conference on Simulation and Modeling, Trondheim, Norway, October 13-14, 2005. 

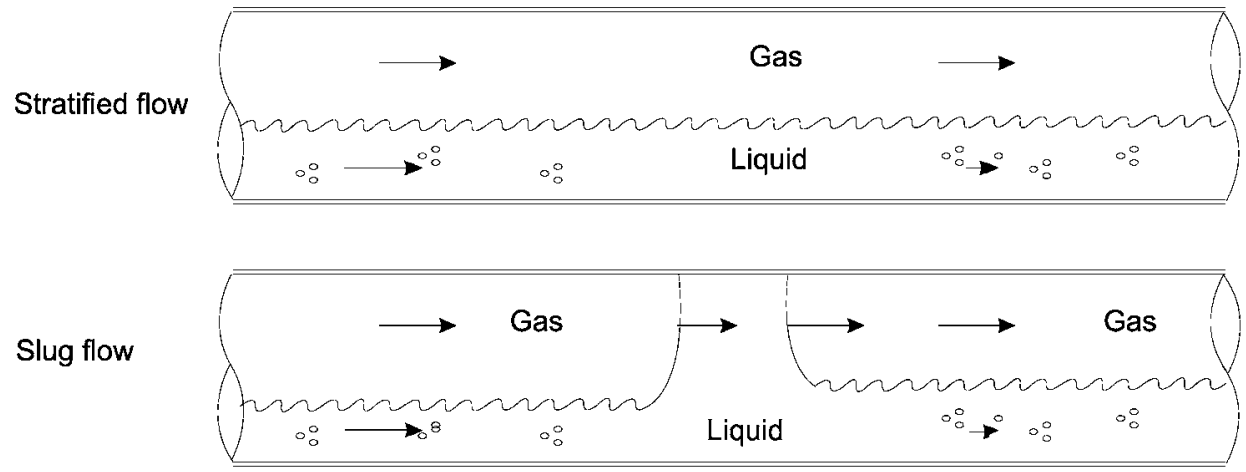

Figure 1. Figure showing the stratified and slug flow regimes.

lengths of more than 500 pipe diameters forming in horizontal pipelines were first observed in the Alaskan Prudhoe Bay-field (Brill, Schmidt, Coberly, Herring \& Moore, 1981; Scott, Shoham \& Brill, 1989). More recently, long growing slugs have been observed in North Sea field lines (Kristiansen, 2004).

The goal of this article is to examine if active control of growing slugs in horizontal pipelines is feasible and to determine if such slugs can be controlled with realistic actuators and measurements.

This paper is organized as follows. Section 2 investigates the controllability of growing slugs by manipulation of inlet and outlet chokes. Section 3 describes a low-order model of inlet choke-slug growth dynamics for observer design. In section 4 an observer based on the model is developed. Section 5 contains conclusions.

\section{Controllability}

Controllability of slug length by inlet and outlet chokes has been investigated by OLGA2000 ${ }^{1}$-simulations (Bendiksen, Halnes \& Moe, 1991). The PI-controller

$$
u=-K_{p}\left(L_{s}-L_{s, \mathrm{ref}}\right)-K_{i} \int_{t_{0}}^{t}\left(L_{s}-L_{s, \mathrm{ref}}\right) d \tau
$$

is used to calculate the choke opening $u$ to drive the slug length $L_{s}$ to a reference value $L_{s, \text { ref. }}$ Figures 2 and 3 show a comparison of controlled slug length with uncontrolled slug length when (1) is applied to the inlet and outlet chokes respectively. In the OLGA2000simulations, a measurement of slug length $L_{s}$ was available. It is seen that when a measurement or estimate of slug length is available, growing slugs can be driven toward a reference length by manipulating either inlet or outlet chokes.

The gains on slug length were found to depend on slug position. The gains were larger when the slug was close to the choke being manipulated. The gain of the inlet choke on slug length was virtually zero for slugs close to the outlet.

1. Scandpower, version 4.13.1. 

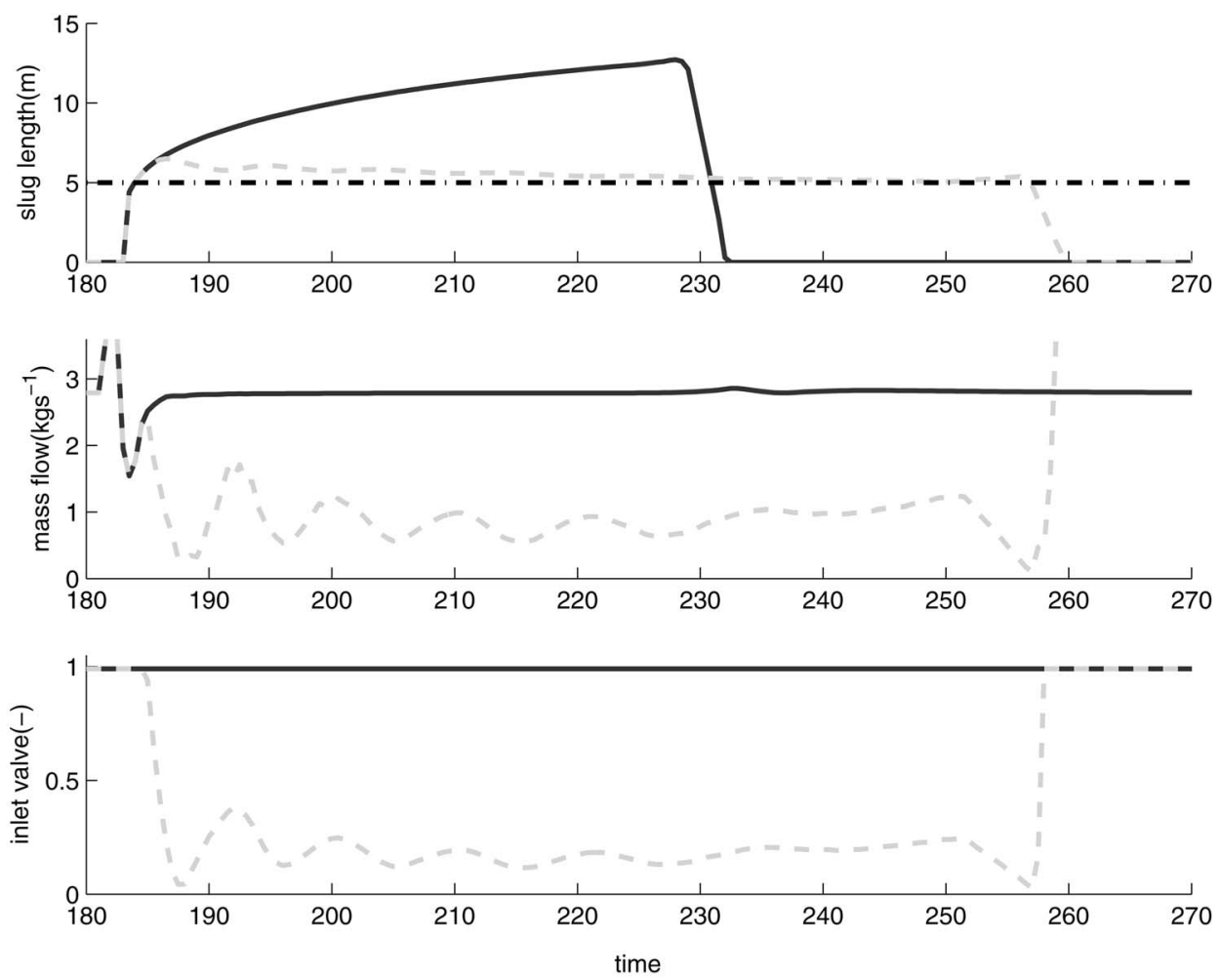

Figure 2. Active control using the inlet choke: A growing slug (solid) compared with a slug controlled by (1) (dashed) toward a reference length (dashdot).

\subsection{Discussion}

In (1) slug length is controlled directly with feedback from a slug length measurement. Inlet pressure tends to grow with slug length, so slug length may also be controlled indirectly with feedback from an inlet pressure measurement. The motivation for investigating direct control of slug length is the possible benefits of expressing slug suppression in terms of a set point on slug length rather than inlet pressure.

Partially closing chokes to reduce slug length is a trade-off between the advantages of steadier flow conditions at the outlet against the costs of decreased production due to choking. Simulations have shown that the gains of inlet and outlet chokes on slug length may be small for some slug positions. Advanced slug length controllers might benefit from a slug position measurement to avoid decreasing production when the advantages of choking are slight.

\section{A model of inlet choke-slug growth dynamics}

The above discussion highlights the utility of a slug length and -position measurement. Such measurements are not commonly available in offshore pipelines. This motivates the design of a slug length- and position observer. This will be the focus of the rest of this paper. In this section, a model which can be used in the synthesis of such an observer is developed. 

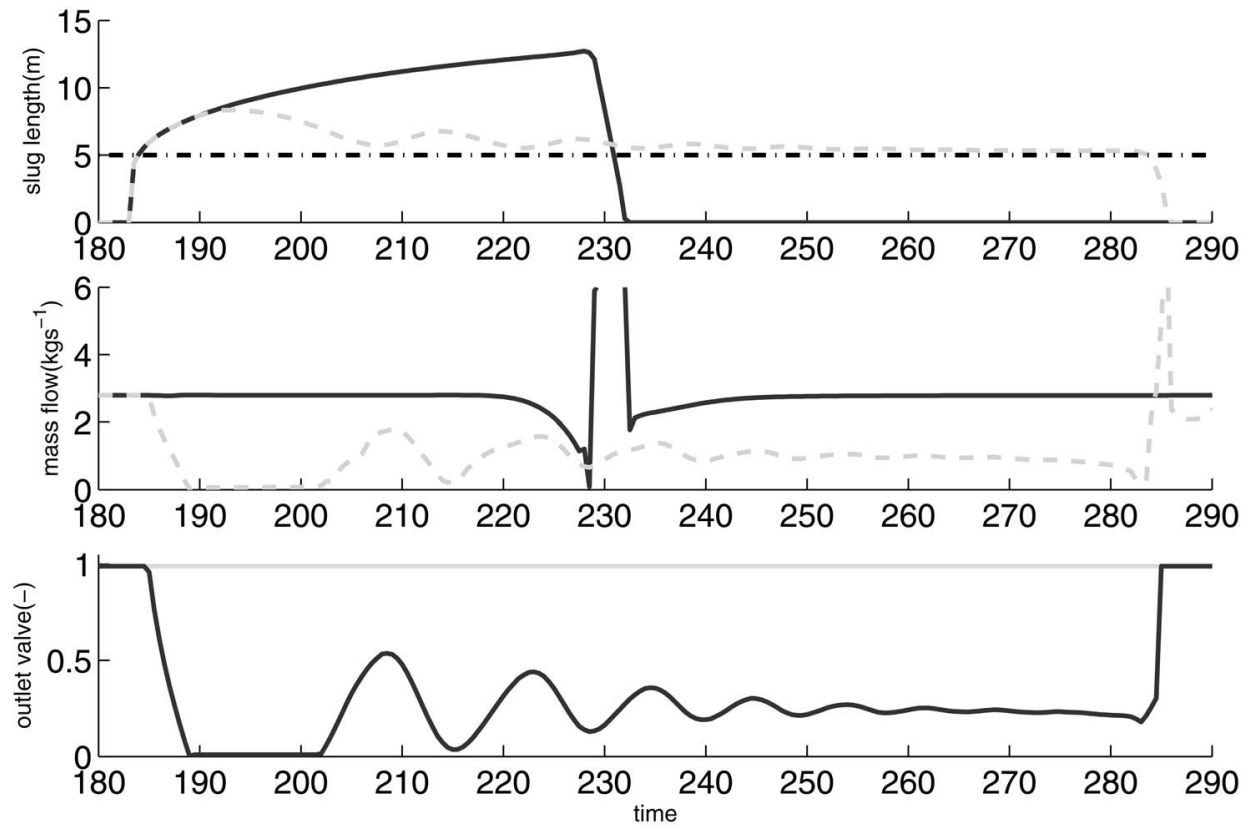

Figure 3. Active control using the outlet choke: A growing slug (solid) compared with a slug controlled by (1) (dashed) toward a reference length (dashdot).

\subsection{Modelling}

In stratified flows such as in Figure 1, gas moves above a liquid layer. Interfacial shear forces between the phases accelerate the liquid, which moves at a significantly lower velocity than the gas phase. When a slug forms in a horizontal pipeline, the blockage caused by the slug causes gas to accumulate upstream of the slug. This accumulation causes an increase in upstream pressure and accelerates the slug and the liquid in it. Due to the turbulent mixing at the slug front, some gas is entrained in the slug. Since the slug body moves quicker than the liquid in front of it, liquid is scooped up at the slug front as the slug moves forward. The liquid moving in the slug body travels at a higher velocity than in the stratified layers, but moves at a slower velocity than the slug itself, causing the slug to shed liquid at its tail (Taitel \& Dukler, 1976).

The objective of this section is to model the dynamics of a growing slug and relate it to choke dynamics. The model should be simple and of low order so as to be suitable for observer design. For simplicity, only the inlet choke will be included in the model.

Modelling is based on applying mass- and impulse balances to the five control volumes shown in Figure 7.

Simplifying assumptions are made to reduce model complexity. Energy balances and phase changes are neglected. Speeds and holdups are assumed to be homogeneous in each control volume. The pipeline is assumed to be perfectly horizontal and to include only a single slug. Droplets in the gas flow and gas entrainment in the stratified liquid films are neglected. No balance is kept for gas entrainment in the slug. The slug is modelled as a mixture with constant average holdup and average density. Holdup and gas density downstream of the slug is assumed constant. The pressure at the outlet is assumed constant. 

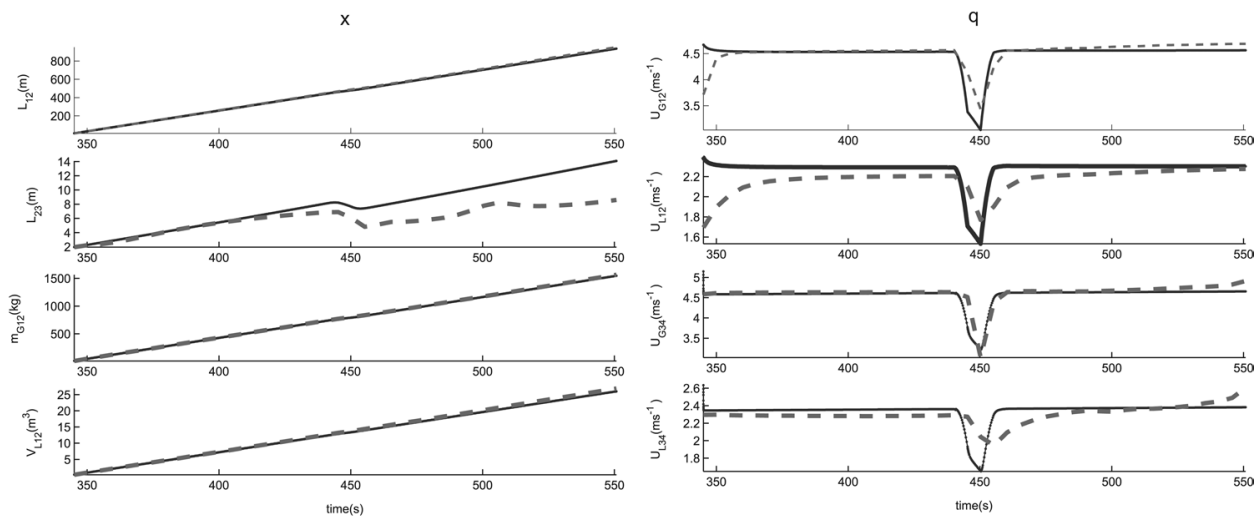

Figure 4. Model verification against OGLA2000: $\mathbf{x}$ and $\mathbf{q}$ (solid) compared with $\mathbf{x}_{e s t}$ and $\mathbf{q}_{\text {est }}$ (dashed).

The complete model (29)-(36) is given in Appendix A. The model can be written on the form (2)-(3), a semi-explicit differential-algebraic equation system, with states (4) and algebraic variables (5).

$$
\begin{aligned}
\dot{\mathbf{x}} & =\mathbf{f}(\mathbf{x}, \mathbf{q}, \mathbf{u}) \\
\mathbf{0} & =\mathbf{g}(\mathbf{x}, \mathbf{q}, \mathbf{u}) \\
\mathbf{x} & =\left[\begin{array}{llll}
L_{12} & L_{23} & m_{G 12} & V_{L 12}
\end{array}\right]^{T} \\
\mathbf{q} & =\left[\begin{array}{llll}
U_{G 12} & U_{L 12} & U_{G 34} & U_{L 34}
\end{array}\right]^{T}
\end{aligned}
$$

\subsection{Verifying the model}

The model was verified against OLGA2000 and experiments. The slug-tracking module of OLGA2000 was used to avoid numerical diffusion at the slug front and tail.

Friction factors were tuned by a least-squares approach to make pressure drops equations and the holdup equation match OLGA2000 as closely as possible.

Figure 4 shows a comparison of a simulation of the model and OLGA2000 when a ramp down followed by a ramp up is applied to the inlet choke. The simulated system is here a one kilometer long 12 inch pipeline at 48 bar with natural gas as the gas phase and and gas condensate as the liquid phase. The model is seen to agree well with OLGA2000. The discrepancy in slug length seen in Figure 4 is caused by numerical issues with OLGA2000 which prevented the holdup downstream of the slug from being totally even, as is assumed in the model. Algebraic states appear to diverge from the model as the slug front approaches the end of the pipeline and the model breaks down when the slug front leaves the pipeline. These deviations are not consequential for control.

Figure 5 shows a comparison of the model with experimental data from the SINTEF Medium Scale Three-Phase Flow Loop ${ }^{2}$. The system was a 103 meter long near-horizontal pipeline at atmospheric pressure with inner diameter of 0.069 meters, air as the gas phase and oil (Exxol D80) as the liquid phase. No phase speed measurements were 

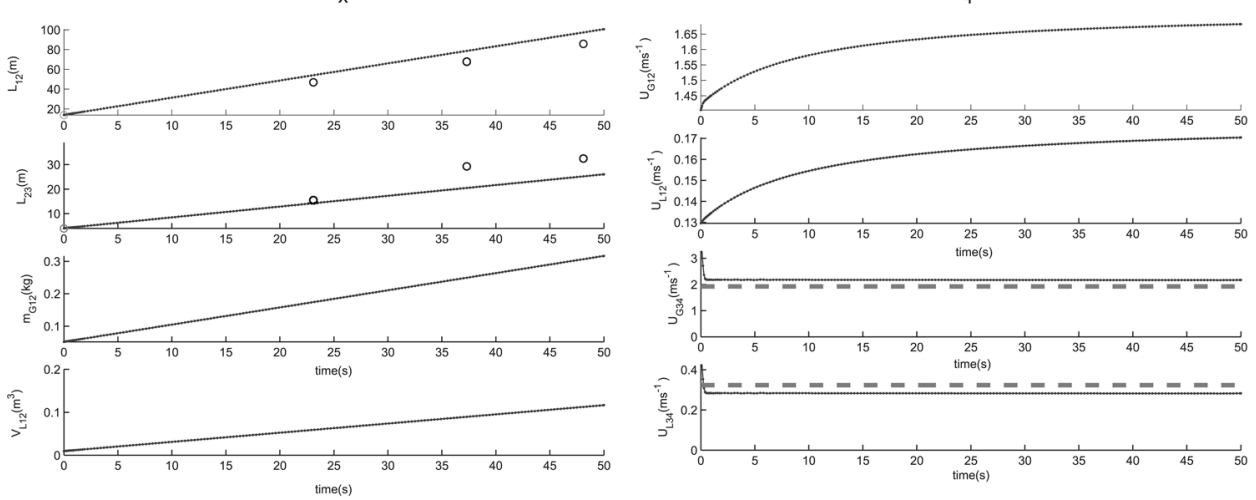

Figure 5. Model verification against SINTEF Medium Scale Three-Phase Flow Loop: $\mathbf{x}$ and $\mathbf{q}$ (solid) compared with $\mathbf{x}_{e s t}$ and $\mathbf{q}_{e s t}$ (dashed).

available, and the slug length and position were deduced from holdup-measurements at four points along the pipeline. The model is seen to agree well with experiments.

\section{Observability and observer design}

Measurement configurations which make the linearized, tuned model observable around realistic trajectories can be found with the observability theory for linear systems. A Luenberger observer is designed to test if the suggested measurement configuration can yield good state estimates in practice.

\subsection{Linearization}

Observability analysis and observer design is based on a linearization of the model. The procedure is similar to Becerra, Roberts \& Griffiths (2001). A first-order Taylorexpansion around the trajectory $(\overline{\mathbf{x}}, \overline{\mathbf{q}}, \overline{\mathbf{u}})$ is performed. Letting $(\tilde{\mathbf{x}}, \tilde{\mathbf{q}}, \tilde{\mathbf{u}})$ denote the offset from the trajectory, so that $\overline{\mathbf{x}}=\mathbf{x}-\tilde{\mathbf{x}}, \overline{\mathbf{q}}=\mathbf{q}-\tilde{\mathbf{q}}, \overline{\mathbf{u}}=\mathbf{u}-\tilde{\mathbf{u}}$ :

$$
\begin{aligned}
& \dot{\mathbf{x}}=\mathbf{f}(\tilde{\mathbf{x}}+\tilde{\mathbf{x}}, \tilde{\mathbf{q}}+\overline{\mathbf{q}}, \tilde{\mathbf{u}}+\overline{\mathbf{u}})=\mathbf{f}(\overline{\mathbf{x}}, \overline{\mathbf{q}}, \overline{\mathbf{u}})+\mathbf{F}_{x} \tilde{\mathbf{x}}+\mathbf{F}_{u} \tilde{\mathbf{u}}+\mathbf{F}_{q} \tilde{\mathbf{q}} \\
& \mathbf{0}=\mathbf{g}(\tilde{\mathbf{x}}+\overline{\mathbf{x}}, \tilde{\mathbf{q}}+\overline{\mathbf{q}}, \tilde{\mathbf{u}}+\overline{\mathbf{u}}) \mathbf{g}(\overline{\mathbf{x}}, \overline{\mathbf{q}}, \overline{\mathbf{u}})+\mathbf{G}_{x} \tilde{\mathbf{x}}+\mathbf{G}_{u} \tilde{\mathbf{u}}+\mathbf{G}_{q} \tilde{\mathbf{q}}
\end{aligned}
$$

where $\mathbf{F}_{x}, \mathbf{F}_{q}, \mathbf{F}_{u}$ and $\mathbf{G}_{x}, \mathbf{G}_{q}$ and $\mathbf{G}_{u}$ are Jacobi-matrices of $\mathbf{f}$ and $\mathbf{g}$ respectively.

Since $\dot{\overline{\mathbf{x}}}=\mathbf{f}(\overline{\mathbf{x}}, \overline{\mathbf{q}}, \overline{\mathbf{u}})$ and $\mathbf{g}(\overline{\mathbf{x}}, \overline{\mathbf{q}}, \overline{\mathbf{u}})=0$,

$$
\begin{aligned}
& \dot{\tilde{\mathbf{x}}}=\mathbf{F}_{x} \tilde{\mathbf{x}}+\mathbf{F}_{u} \tilde{\mathbf{u}}+\mathbf{F}_{q} \tilde{\mathbf{q}} \\
& \mathbf{0}=\mathbf{G}_{x} \tilde{\mathbf{x}}+\mathbf{G}_{u} \tilde{\mathbf{u}}+\mathbf{G}_{q} \tilde{\mathbf{q}} .
\end{aligned}
$$

For the model developed, $\mathbf{G}_{q}$ is non-singular. Then

$$
\tilde{\mathbf{q}}=-\mathbf{G}_{q}^{-1}\left[\mathbf{G}_{x} \tilde{\mathbf{x}}+\mathbf{G}_{u} \tilde{\mathbf{u}}\right] .
$$

Substituting (10) into (8) gives

$$
\dot{\tilde{\mathbf{x}}}=\mathbf{A} \tilde{\mathbf{x}}+\mathbf{B} \tilde{\mathbf{u}},
$$

where

$$
\mathbf{A} \stackrel{\text { def }}{=} \mathbf{F}_{q} \mathbf{G}_{q}^{-1} \mathbf{G}_{x}-\mathbf{F}_{x}
$$




$$
\mathbf{B} \stackrel{\text { def }}{=} \mathbf{F}_{q} \mathbf{G}_{q}^{-1} \mathbf{G}_{u}-\mathbf{F}_{u}
$$

The measurement equation $\mathbf{y}=\mathbf{h}(\mathbf{x}, \mathbf{q}, \mathbf{u})$ to be determined relates measured variables to the internal variables of the model. In a similar fashion to (11), the linearized measurement equation

$$
\tilde{\mathbf{y}}=\mathbf{C} \tilde{\mathbf{x}}+\mathbf{D} \tilde{\mathbf{u}}
$$

can be found, where

$$
\begin{aligned}
& \mathbf{C} \stackrel{\text { def }}{=} \mathbf{H}_{q} \mathbf{G}_{q}^{-1} \mathbf{G}_{x}-\mathbf{H}_{x} \\
& \mathbf{D} \stackrel{\text { def }}{=} \mathbf{H}_{q} \mathbf{G}_{q}^{-1} \mathbf{G}_{u}-\mathbf{H}_{u}
\end{aligned}
$$

and $\mathbf{H}_{x}$ and $\mathbf{H}_{q}$ are Jacobi-matrices of $\mathbf{h}$.

\subsection{Observer design}

A measurement configuration which renders the linearized model observable can now be found using linear observability theory (Chen, 1999). One such measurement configuration is

$$
\mathbf{y}=\left[\begin{array}{lllll}
p_{1} & U_{G 12} & U_{L 12} & U_{G 34} & U_{L 34}
\end{array}\right]^{T} .
$$

$p_{1}$ is a pressure measurement at the inlet, which is related to $\mathbf{x}$ and $\mathbf{q}$ by (37), given in Appendix B. None of the entires in (17) require mid-pipeline measurements, as $p_{1}, U_{L 12}$ and $U_{G 12}$ can be measured at the inlet and $U_{L 34}$ and $U_{G 34}$ can be measured at the outlet.

An extended-Luenberger observer (Zeitz, 1987) on the form (18)-(19) was designed to verify that the measurements (17) can indeed produce estimates of slug length.

$$
\begin{aligned}
\dot{\mathbf{x}}_{e s t} & =\mathbf{f}\left(\mathbf{x}_{e s t}, \mathbf{q}_{e s t}, \mathbf{u}\right)+\mathbf{K}\left(\mathbf{y}-\mathbf{y}_{e s t}\right) \\
\mathbf{0} & =\mathbf{g}\left(\mathbf{x}_{e s t}, \mathbf{q}_{e s t}, \mathbf{u}\right)
\end{aligned}
$$

The output injection gain $\mathbf{K}$ of the injection term $\mathbf{K}\left(\mathbf{y}-\mathbf{y}_{\text {est }}\right)$ is found by pole-placement on (20), a linearization of (18)-(19). $\mathbf{K}$ is chosen so that eig $(\mathbf{A}-\mathbf{K C})<0$ for observer stability and $|\operatorname{eig}(\mathbf{A}-\mathbf{K C})|>10|\operatorname{eig}(\mathbf{A})|$ for good performance.

$$
\dot{\mathbf{x}}_{e s t}=\mathbf{A} \mathbf{x}_{e s t}+\mathbf{B u}+\mathbf{K}\left(\mathbf{y}-\mathbf{y}_{e s t}\right)
$$

\subsection{Simulation trial}

The observer (18)-(19) was tested against a copy of the low-order model. To investigate observer robustness to model uncertainty, each lumped parameter in the model on which the observer was based was given a random pertubation in the region $\pm 30 \%$, with an average lumped parameter error of $13.2 \%$.

The results are shown in Figure 6. In the simulation, the output injection term was updated at each time step. It was found that applying the same injection term through the whole simulation led to instabilities. As seen in Figure 6, the states $\mathbf{x}_{e s t}$ of the model quickly converge toward the states $\mathbf{x}$ of the process even with parameter uncertainty. $\mathbf{q}_{\text {est }}$ and $\mathbf{q}$ do not converge since no output injection terms are applied to the algebraic variables. 

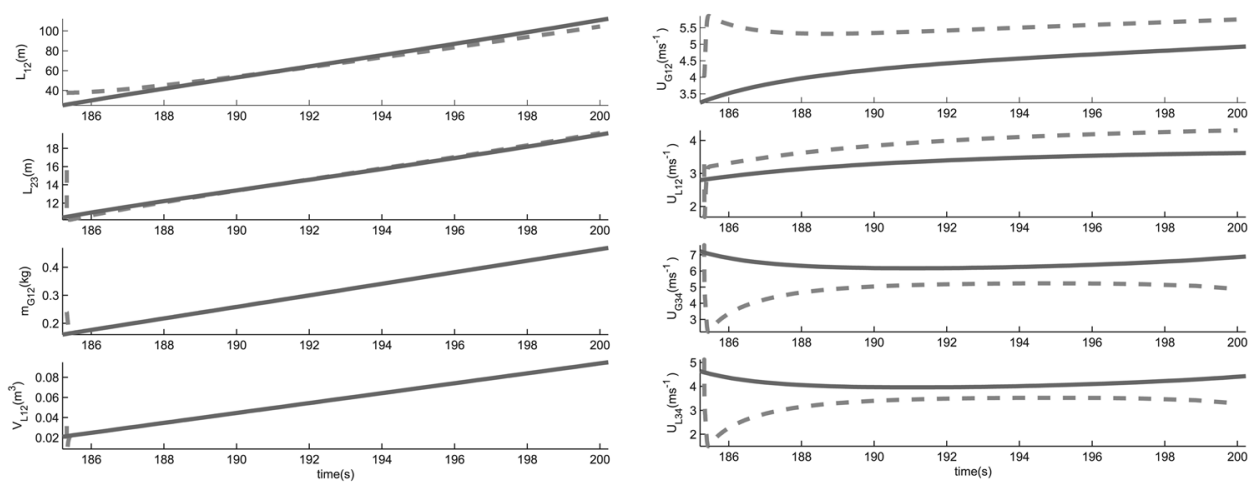

Figure 6. Simulation trial of observer with model uncertainty: $\mathbf{x}$ and $\mathbf{q}$ (solid) compared with $\mathbf{x}_{e s t}$ and $\mathbf{q}_{\text {est }}$ (dashed).

\subsection{Discussion}

Ideally, the observer should be tested against experiments or OLGA2000. Unfortunately, the author did not have experimental data available where every entry in (17) was measured.

A discrepancy between OLGA2000 and experimental data was found. The local upstream pressure $p_{1}$ was found to grow as slug length grows in the experiments, while simulations in OLGA2000 showed no such correlation. This discrepancy prevented verifying the observer against OLGA2000.

\section{Conclusion}

By simulating with OLGA2000 it has been shown that long slugs are controllable by manipulating either inlet or outlet chokes. The design of an observer for slug length and -position was motivated by simulations of the direct control of slug length with a PI-controller. A low-order dynamic model of slug growth and choke dynamics was developed for observer design and found to correspond well to OLGA2000 and experiments.

A measurement configuration which makes the linearized model observable was suggested. The required measurements are rate and pressure measurements at the inlet and outlet. To verify observability in practice, an extended Luenberger observer based on the model was shown to find accurate estimates of slug length and -position, even under model uncertainty.

Further work could focus on testing observer and controller together or on extending the suggested approach to pipelines with multiple slugs. Much further work is also possible on observer design.

The main contribution of this paper is to show that growing slugs are both observable and controllable with actuators and measurements which are available in many existing multiphase pipelines.

\section{Acknowledgments}

The author wishes to thank René Oliemans, TU Delft, for hosting part of my research. The author thanks Olav Kristiansen, SINTEF, for providing experimental data. 


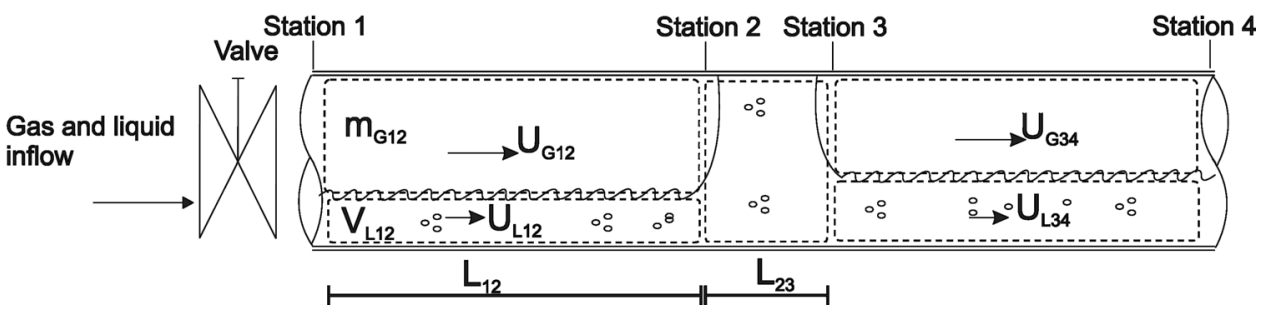

Figure 7. System configuration: inlet choke and pipeline with a slug and two areas of stratified flow, control volumes and independent variables shown.

The author thanks John-Morten Godhavn, Statoil, Ole Morten Aamo, NTNU, Bjarne Foss, NTNU, and Gisle Otto Eikrem, Statoil, for interesting feedback. The author thanks Scandpower for providing OLGA2000.

\section{A. Equations of the low-order model}

\section{A.1. Nomenclature}

All numbered suffixes refer to stations 1-4 shown in Figure 7. Suffices with a single-number refer to the value at a particular station, while double-numbered suffices refer to the values in control volumes between two stations.

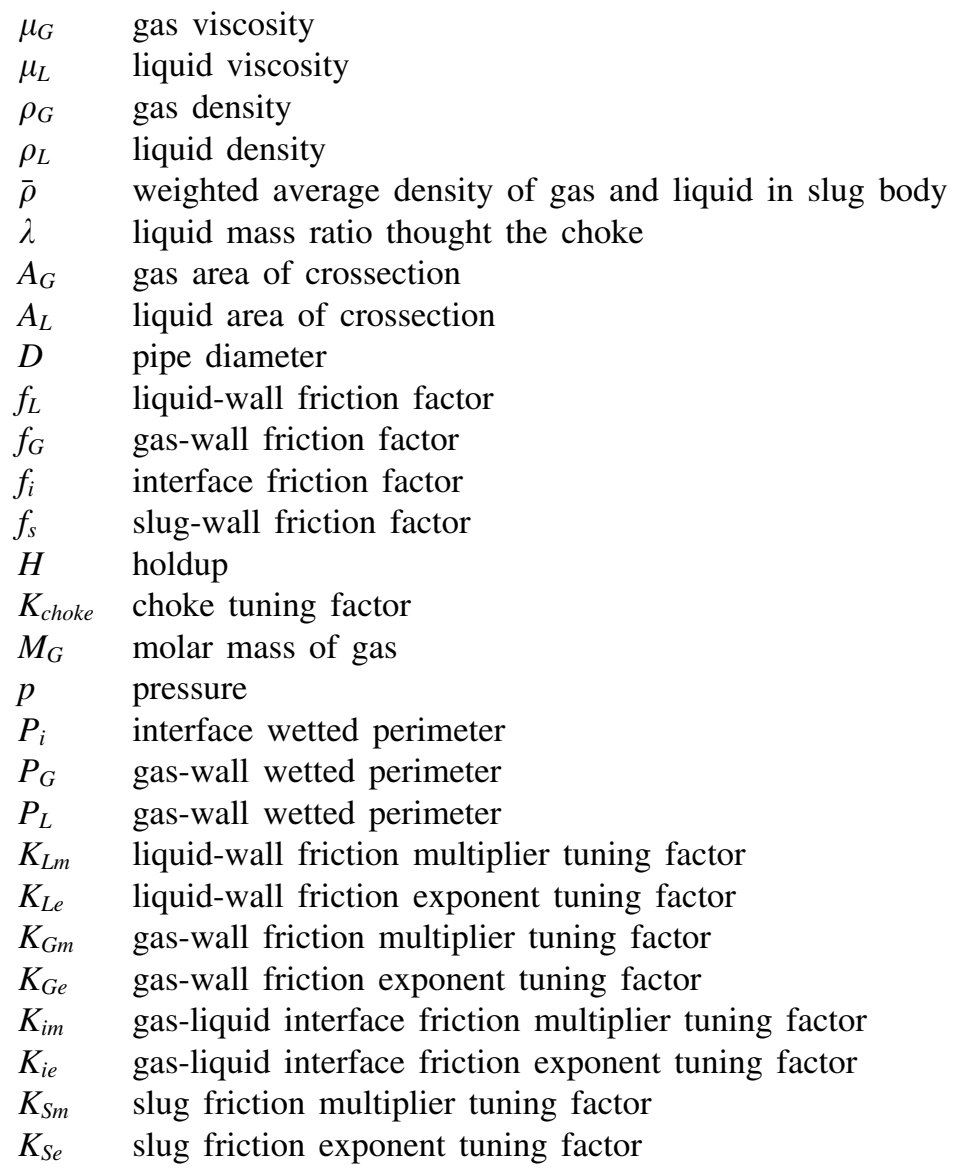


$L_{12} \quad$ position of slug tail

$L_{23} \quad$ slug length

$Q \quad$ volumetric flow

$s \quad$ slip ratio $\frac{U_{G 23}}{U_{L 23}}$

$u \quad$ normalized choke opening

$U_{G} \quad$ gas speed

$U_{L} \quad$ liquid speed

$V_{L} \quad$ volume of liquid

\section{A.2. Model}

The tail of the slug is modeled as a Taylor-bubble (Benjamin, 1968), so that the volumetric flow out the slug tail, $Q_{2}$, is given by

$$
Q_{2}=0.542 A \sqrt{g D} \text {. }
$$

By equating a mass-balance between the upstream stratified layer and the slug tail, as suggested in Woods \& Hanratty (1996), the speed of liquid in the slug $U_{L 23}$ is related to the speeds of the upstream straitifed flow and an empirical slip ratio $s \stackrel{\text { def }}{=} \frac{U_{G 23}}{U_{L 23}}$ as

$$
U_{L 23}=\frac{U_{G 12}\left(1-H_{12}\right)+U_{L 12} H_{12}}{1+(s-1)\left(1-H_{23}\right)} .
$$

The velocity $\dot{L}_{12}$ is described by an impulse balance (29) over the slug body. The pressure acting on the slug tail is modelled by the ideal gas law

$$
p_{2}=\rho_{G 12} \frac{R T}{M_{G}}
$$

to account for compressibility effects.

The dynamics of slug length $\dot{L}_{23}$ is described by a mass balance (30) over the slug body.

The mass flow through the inlet choke is modelled by

$$
\dot{m}=K_{\text {choke }}\left(1-(1-u)^{2}\right),
$$

where $K_{\text {choke }}$ is an empirical fitting factor and $u$ is the normalized choke opening. $\lambda$, theliquid mass ratio thought the choke, is assumed constant. Mass balances in combination with (21) and (24) yield (31) and (32).

The upstream gas velocity $U_{G 12}$ is related to the mass gas flow by (33). The downstream gas velocity $U_{G 34}$ is assumed to be equal to the speed of the slug front (35).

The liquid velocities of the stratified films, $U_{L 12}$ and $U_{L 34}$, are related to the gas velocities $U_{G 12}$ and $U_{G 34}$ by the holdup equations (34) and (36). The holdup equations are found by combining steady-state impulse balances for the gas and liquid layers by eliminating pressure drop.

The model includes four friction factors: the interface friction $f_{i}$, the gas-wall friction $f_{G}$ and the liquid-wall friction $f_{L}$ of the stratified flow regions as well as the slug-wall friction $f_{S}$. These friction factors vary with flow conditions and are normally fitted empirically to data as a function of the Reynolds number. The friction factors for the stratified flows were modelled by

$$
f_{L}=K_{L m}\left(\frac{\rho_{L} U_{L} 4 A_{L}}{\mu_{L} P_{L}}\right)^{-K_{L e}}
$$




$$
\begin{aligned}
f_{G} & =K_{G m}\left(\frac{\rho_{G} U_{G} 4 A_{G}}{\mu_{G}\left(P_{G}+P_{i}\right)}\right)^{-K_{G e}} \\
f_{i} & =K_{i m}\left(\frac{\rho_{G}\left(U_{G}-U_{L}\right) 4 A_{G}}{\mu_{G}\left(P_{G}+P_{i}\right)}\right)^{-K_{i e}},
\end{aligned}
$$

where $K_{L m}, K_{L e}, K_{G m}, K_{G e}, K_{i m}, K_{i e}$, are tuning parameters, and the relations in large parenthesis are Reynolds numbers with hydraulic diameters inserted. The slug friction was modelled as

$$
f_{s}=K_{S m}\left(\frac{D \bar{\rho}\left(U_{L 23} H_{23}+U_{G 23}\left(1-H_{23}\right)\right)}{\mu_{L}}\right)^{-K_{S e}} .
$$

$P_{G}, P_{L}$ and $P_{i}$ are the wetted perimeters illustrated in Figure 8.

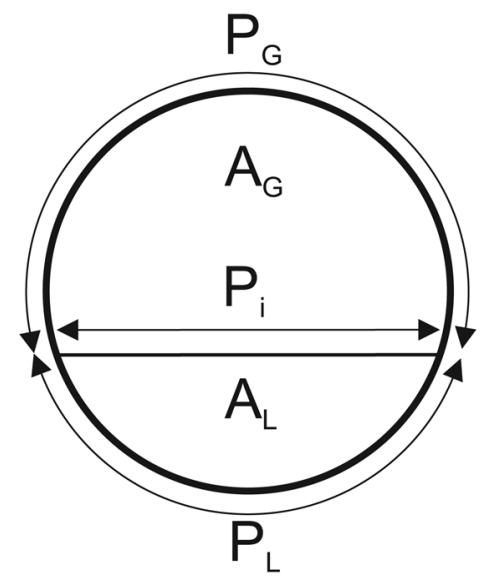

Figure 8. Illustration of the wetted perimeters $P_{L}, P_{G}$ and $P_{i}$ and the phase areas $A_{L}$ and $A_{G}$ in a pipe crossection.

$$
\begin{aligned}
p_{2} & -p_{4}-0.5 \rho_{L} f_{L 34} U_{L 34}^{2} P_{L 34}\left(L-L_{12}-L_{23}\right) \\
- & 0.5 \rho_{G 34} f_{G 34} U_{G 34}^{2} P_{G 34}\left(L-L_{12}-L_{23}\right) \\
- & 0.5 \bar{\rho} f_{s} U_{L 23}^{2} P_{23} L_{23}=\rho_{L}\left(U_{L 34}-\dot{L}_{12}\right) U_{L 34} H_{34} \\
- & \rho_{L} U_{L 23}\left(U_{L 23}-\dot{L}_{12}\right) H_{23} \\
\dot{L}_{23}= & \frac{1}{A}\left[A_{L 34}\left(\dot{L}_{12}-U_{L 34}\right)-Q_{2}\right] \\
\dot{m}_{G 12}= & (1-\lambda) K_{\text {choke }}\left(1-(1-u)^{2}\right) \\
\dot{V}_{L 12}= & \frac{\lambda K_{\text {choke }}\left(1-(1-u)^{2}\right)}{\rho_{L}}+Q_{2} \\
U_{G 12}= & \left(\frac{1}{1-H_{12}}\right) \frac{\dot{m}_{G 12}}{\rho_{G 12} A} \\
0= & \frac{1}{2} f_{G 12} \rho_{G 12} U_{G 12}^{2} P_{G 12} \frac{1}{A_{G 12}}-\frac{1}{2} f_{L 12} \rho_{L} U_{L 12}^{2} P_{L 12} \frac{1}{A_{L 12}} \\
& +\frac{1}{2} f_{i 12} \rho_{G 12}\left(U_{G 12}-U_{L 12}\right)^{2} P_{i 12}\left(\frac{1}{A_{L 12}}+\frac{1}{A_{G 12}}\right)
\end{aligned}
$$




$$
\begin{aligned}
U_{G 34}= & \dot{L}_{12}+\dot{L}_{23} \\
0= & \frac{1}{2} f_{G 34} \rho_{G 34} U_{G 34}^{2} P_{G 34} \frac{1}{A_{G 34}}-\frac{1}{2} f_{L 34} \rho_{L} U_{L 34}^{2} P_{L 34} \frac{1}{A_{L 34}} \\
& +\frac{1}{2} f_{i 34} \rho_{G 34}\left(U_{G 34}-U_{L 34}\right)^{2} P_{i 34}\left(\frac{1}{A_{L 34}}+\frac{1}{A_{G 34}}\right)
\end{aligned}
$$

\section{B. Measurement equation}

The measurement equation (37) relates the pressure at the inlet $p_{1}$ to the algebraic variables $\mathbf{q}$ and the states $\mathbf{x}$.

$$
p_{1}=p_{4}+\left(\frac{d p}{d x}\right)_{12} L_{12}+\left(\frac{d p}{d x}\right)_{23} L_{23}+\left(\frac{d p}{d x}\right)_{34}\left(L-L_{12}-L_{23}\right),
$$

where

$$
\begin{aligned}
& \left(\frac{d p}{d x}\right)_{12}=0.5 \rho_{G 12} f_{G 12} U_{G 12}^{2} \frac{P_{G 12}}{A}+0.5 \rho_{L} f_{L 12} U_{L 12}^{2} \frac{P_{L 12}}{A} \\
& \left(\frac{d p}{d x}\right)_{23}=0.5 \bar{\rho} f_{s} U_{L 23}^{2} \frac{P_{23}}{A} \\
& \left(\frac{d p}{d x}\right)_{34}=0.5 \rho_{G 34} f_{G 34} U_{G 34}^{2} \frac{P_{G 34}}{A}+0.5 \rho_{L} f_{L 34} U_{L 34}^{2} \frac{P_{L 34}}{A}
\end{aligned}
$$

\section{References}

Becerra, V., Roberts, P. \& Griffiths, G. (2001). 'Applying the extended kalman filter to systems described by nonlinear differential-algebraic equations', Control Engineering Practice 9, pp. 267-281.

Bendiksen, K., Halnes, D. Moe, R.\& Nuland, S. (1991). 'The dynamic two-fluid model olga: Theory and application', SPE Production Engineering 6, pp. 171-180.

Benjamin, T. (1968). 'Gravity currents and related phenomena', J.Fluid Mech. 89, pp. 209-248.

Brill, J., Schmidt, Z., Coberly, W., Herring, J. \& Moore, D. (1981). 'Analysis of two-phase tests in large diametre prudhoe bay field', SPEJ, pp. 363-378.

Chen, C.-T. (1999). Linear System Theory and Design, 3 edn, Oxford Univ. Press.

Godhavn, J., Fard, M. P. \& Fuchs, P. (2005). 'New slug control strategies, tuning rules and experimental results', Journal of Process Control 15, pp. 547-557.

Havre, K. \& Dalsmo, M. (2001). Active feedback control as the solution to severe slugging, in 'SPE Annual Technical Conference and Exhibition'.

KristiansEN, O. (2004). Experiments on the transition from stratified to slug flow in multiphase pipe flow, Phd-thesis, Norwegian University of Science and Technology (NTNU).

Schmidt, Z., BriLl, J. \& BegGs, H. (1979). 'Choking can eliminate severe pipeline slugging', Oil and Gas Journal, pp. 230-238.

Scott, S., Shoham, O. \& BRILl, J. (1989). 'Prediction of slug length in horizontal, large-diameter pipes', SPE Production Engineering, pp. 335-340.

Skofteland, G. \& Godhavn, J.-M. (2003). Suppression of slugs in multiphase flow lines by active use of topside choke, in 'Multiphase'03 San Remo'.

TAITEL, Y. \& DuKLER, A. (1976). 'A model for predicting flow regime transitions in horizontal and near horizontal gas-liquid flow', AIChE J. 22, pp. 47-55. 
Woods, B. \& HanRatTy, T. (1996). 'Relation of slug stability to shedding rate', Int.J.Multiphase Flow 22, pp. 809-828.

ZeITZ, M. (1987). 'The extended luenberger observer for nonlinear systems', Systems \& Control Letters 9 10, pp. 149-156. 